\title{
Focus on determinants of male fertility
}

\author{
E R S Roldan \\ Reproductive Ecology and Biology Group, Museo Nacional de Ciencias Naturales (CSIC), c/José Gutierrez Abascal 2, \\ 28006 Madrid, Spain
}

Correspondence should be addressed to E R S Roldan; Email: roldane@mncn.csic.es

Understanding the main determinants of male fertility would allow us to advance our knowledge of male reproductive function and lifetime reproductive strategies, and also to design appropriate tests to evaluate males and semen samples collected from them. Sperm evaluation has moved away from subjective analysis to more objective means of assessing cell morphology, dimensions, functions, and the underlying mechanisms that ultimately lead to successful fertilization and generation of viable offspring. Future studies dealing with sperm function as well as biomarker identification and the development of molecular tools will undoubtedly yield better and more reliable assays.

Spermatozoa experience many complex processes and these influence, to varying degrees, the fertilizing capacity of the sperm cell. After differentiation in the testis, spermatozoa undergo several key events towards fertilization; events, which have, to a greater or lesser extent, been identified as potential indicators of sperm quality.

The analysis of male reproductive function needs to consider species and individual variation, and recognize that ejaculate components, including sperm form and function, may vary between ejaculates from the same animal. It is also important to bear in mind that there are important differences between the species in terms of reproductive strategies. Species or populations may differ depending on the selection pressures that have acted on them. In the case of wild populations, selective pressures will differ markedly depending on mating systems and, in particular, the intensity of sperm competition (i.e. competition between rival ejaculates when females mate with more than one male). In the case of domestic species subjected to artificial selection, breeding schemes differ in the traits that they target (e.g. selection for milk yield, meat production, or male fertility), and these differences may have a profound influence on sperm traits. In other species (e.g. man, horses, or companion animals) many studies focus on sub-fertile or infertile patients, and this may generate a bias when trying to understand the potential relationship between semen parameters and sperm fertility.

The identification of sperm parameters important for fertility also requires advances on both the development of laboratory tests for sperm assessment and the definition of the best conditions for fertility evaluation (Amann 1989, Amann \& Hammerstedt 1993). Sperm assessment methods can measure general traits such as concentration, motility, morphology, organelle integrity, and, more recently, DNA integrity (Aitken 2006, Rodriguez-Martinez 2006, 2007). More complex methods aim to evaluate various aspects of sperm function that may be important during the journey to the oocyte (e.g. penetration of female tract barriers, hyperactivation, capacitation, or acrosomal exocytosis; Aitken 2006). On the other hand, fertility can be tested in vitro (e.g. by in vitro fertilization) or in vivo (e.g. by artificial insemination). The latter, restricted to domestic species, should involve a minimum number of females to minimize individual variation and environmental effects (Amann 1989, Amann \& Hammerstedt 1993).

Few tests for semen evaluation have incorporated molecular analyses of sperm function and few have attempted to link this information with fertility (Lefièvre et al. 2007). In the future it will be important to identify cellular and molecular markers that reveal various aspects of the potential of sperm to undergo functional changes. Thus, it will be most rewarding to explore, under standardized conditions, changes in protein phosphorylation, lipid signaling pathways, or $\mathrm{Ca}^{2+}$ regulation. Future tests can perhaps also incorporate proteomic investigations that would help identify those proteins associated with sperm function, thus revealing differences between fertile and sub-fertile individuals (Lefièvre et al. 2007).

It is becoming clear that measures of single sperm traits, or the results of single functional tests, are poorly correlated with fertility (Rodriguez-Martinez 2007). As a result, the emphasis now is on analyses that incorporate multiple variables to examine how different sperm parameters interact to determine fertility.

The majority of investigations dealing with sperm function have concentrated either on in vivo studies or on the development of laboratory methods that mimic conditions found in the female tract. However, transport and selection in the female tract is only one of the two major selective forces that have molded sperm cells in species with internal fertilization. Sperm competition, the 
other selective force, is now recognized as an important factor that may have shaped semen characteristics (Roldan et al. 1992) and sperm function (Gomendio et al. 2006). Although there is now ample evidence for sperm competition in mammals, including domestic species, there appears to have been little attempt at understanding this selective force. Humans, on the other hand, show little evidence of sperm competition (Gomendio et al. 1998) but, nevertheless, may prove useful to develop some in vitro tests using this principle.

The three reviews included in this issue address different scenarios for the characterization of determinants of male fertility. They concentrate on natural populations, domestic species, and humans. The first review (Gomendio et al. 2007) highlights male reproductive strategies and draws attention to the enormous variation in sperm traits found between individuals in natural populations. This variation in semen traits has important implications for male fertility. Under these conditions it has been possible to identify which semen traits are the main determinants of fertility. Differences between males in fertility rates are much greater than expected and suggest that semen traits influence male reproductive success to a large extent. Thus, sperm traits are under strong selection pressure in natural populations.

The review by Petrunkina et al. (2007) emphasizes the need to understand processes, and their underlying mechanisms, in the life of the fertilizing spermatozoon. It focuses on the capacity of spermatozoa to regulate cellular volume, the interactions between sperm and oviductal cells, and the assessment of capacitation in vitro. Preliminary results from tests assessing the link between these processes and fertility look promising. These authors also stress that subfertility may relate to a suboptimal response (i.e. either too high or too low) rather than to a lack of or poor response.

Finally, Lewis (2007) addresses issues related to the evaluation of human spermatozoa. She highlights the limitations of conventional parameters and points to the fact that assisted reproductive techniques, such as intracytoplasmic sperm injection, have diverted attention away from the development of more relevant measures of sperm function. This review also points out that, although news tests have been developed in recent years, few if any have found their way into routine use. More importantly, the task of identifying a set of practical and cost-effective tests is strongly endorsed.

When examining the themes covered by these reviews, some important issues emerge. First, simple, basic sperm parameters may be very informative in species from natural populations. In contrast to the situation in domestic animals, such semen parameters appear to relate well to male fertility. This could be due to the existence of strong selective pressures to improve male reproductive success in wildlife, and a higher variability in sperm traits and male fertility in natural populations. This emphasizes the need to better characterize male fertility in natural populations and to design adequate experiments to explore relationships between sperm traits and fertility. Secondly, there are differences in the main sperm evaluation tests used for domestic animals and humans. It is not entirely clear why this should be, but it may be related to how sperm cells are used in assisted reproductive technologies. The extensive use of artificial insemination in domestic species (with or without cryopreserved spermatozoa) has led to the development of tests to evaluate sperm survival, metabolism, and premature or altered capacitation. In humans, where in vitro fertilization or intracytoplasmic sperm injection (ICSI) are practiced, emphasis appears to be on structural and acrosomal integrity and, more importantly, on sperm-oocyte interactions, including acrosomal exocytosis. Furthermore, information on DNA fragmentation, its relevance for post-fertilization development, and studies on the negative effects of reactive oxygen species, also predominate in human sperm evaluation.

The reviews contained in this issue are based on presentations delivered at the opening session of the 10th International Symposium on Spermatology, which took place in Madrid in September 2006. This session was sponsored by the Society for Reproduction and Fertility.

\section{References}

Aitken RJ 2006 Sperm function tests and fertility. International Journal of Andrology 29 69-75.

Amann RP 1989 Can the fertility potential of a seminal sample be predicted accurately? Journal of Andrology 10 89-98.

Amann RP \& Hammerstedt RH 1993 In vitro evaluation of sperm quality. An opinion. Journal of Andrology 14 397-406.

Gomendio M, Harcourt AH \& Roldan ERS 1998 Sperm competition in mammals. In Sperm Competition and Sexual Selection, pp 667-751. Eds TR Birkhead \& AP Møller. London: Academic Press.

Gomendio M, Martin-Coello J, Crespo C, Magana C \& Roldan ERS 2006 Sperm competition enhances functional capacity of mammalian spermatozoa. PNAS 103 15113-15117.

Gomendio M, Malo AF, Garde J \& Roldan ERS 2007 Sperm traits and male fertility in natural populations. Reproduction 134 19-29.

Lefièvre L, Bedu-Addo K, Conner SJ, Machado-Oliveira GSM, Chen Y, Kirkman-Brown JC, Afnan MA, Publicover SJ, Ford WCL \& Barratt CLR 2007 Counting sperm does not add up any more: time for a new equation? Reproduction 133 675-684.

Lewis SEM 2007 Is sperm evaluation useful in predicting human fertility? Reproduction 134 31-40.

Petrunkina AM, Waberski D, Günzel-Apel AR \& Töpfer-Petersen E 2007 Determinants of sperm quality and fertility in domestic species. Reproduction 134 3-17.

Rodriguez-Martinez H 2006 Can we increase the estimative value of semen assessment? Reproduction in Domestic Animals $4 \mathbf{1}$ (Supplement 2) 2-10.

Rodriguez-Martinez H 2007 State of the art in farm animal sperm evaluation. Reproduction, Fertility, and Development 19 91-101.

Roldan ERS, Gomendio M \& Vitullo AD 1992 The evolution of eutherian spermatozoa and underlying selective forces: female selection and sperm competition. Biological Reviews of the Cambridge Philosophical Society 67 551-593. 